\title{
Phenotypes of major immediate-early gene mutants of mouse cytomegalovirus
}

\author{
Andreas Busche • Ana Angulo • Penelope Kay-Jackson • \\ Peter Ghazal $\cdot$ Martin Messerle
}

Received: 27 November 2007 / Published online: 1 February 2008

(C) Springer-Verlag 2008

\begin{abstract}
Immediate-early (IE) genes are the first genes to be transcribed during the lytic replication cycle of cytomegaloviruses (CMV), and encode nonstructural proteins, which are assumed to have mainly regulatory functions. The IE proteins may play important roles in the pathogenesis of CMV in vivo, for instance during the establishment of latency and during reactivation. We constructed mouse CMV mutants with disruptions in the major IE genes, ie 1 and ie3, to study the roles of these genes in the context of the viral infection. Here we summarize the current results on the characterization of these mutants and give a perspective of the future research in this field.
\end{abstract}

Keywords Mouse cytomegalovirus $\cdot$ Immediate-early genes $\cdot$ Viral mutants $\cdot$ Phenotypes

\section{Introduction}

Upon infection of cells with cytomegaloviruses (CMV), viral gene expression starts with the transcription of immediate-early (IE) genes. Expression of these genes is activated by cellular transcription factors and virion-associated

A. Busche $\cdot$ P. Kay-Jackson · M. Messerle $(\varangle)$ Department of Virology, Hannover Medical School, Carl-Neuberg-Str. 1, 30625 Hannover, Germany e-mail: messerle.martin@mh-hannover.de

\author{
A. Angulo \\ Institut d'Investigacions Biomédiques August Pi I Sunyer, \\ 08036 Barcelona, Spain \\ P. Ghazal \\ Division of Pathway Medicine, University of Edinburgh, \\ Edinburgh EH16 4SB, UK
}

regulatory proteins and is thus independent of de novo synthesis of any viral proteins [1]. It is assumed that the IE proteins represent mainly regulatory proteins, which participate in the activation of viral genes needed later in the infection cycle, thereby promoting lytic replication of CMV. The major IE region of mouse CMV (MCMV) displays a high degree of structural similarity to the corresponding genomic region of human CMV (HCMV). A transcription unit composed of five exons is located downstream of a complex regulatory element consisting of enhancer elements and the major immediate early promoter (MIEP), and gives rise to at least two alternatively spliced major IE transcripts (Fig. 1). One transcript consisting of exons 1-4 encodes the IE1 protein and the other transcript composed of exons 1, 2, 3 and 5 encodes the IE3 protein in MCMV (or the homologous IE2 protein in HCMV). The $89 \mathrm{kDa}$ IE1 protein of MCMV consists of 595 amino acids (aa) and the $72 \mathrm{kDa}$ IE1 protein of HCMV of 491 aa. Additional proteins originate from the IE1 regions of MCMV and HCMV, e.g. the pp76 modification product of the $89 \mathrm{kDa}$ IE1 protein or a $19 \mathrm{kDa}$ protein of HCMV. We have therefore to consider that the functions ascribed to the major IE1 products may at least in part be due to these other IE1 proteins. An alignment of the amino acid sequences of the MCMV and HCMV IE1 proteins is possible when the conditions specified in the legend of Fig. 2 are applied. The alignment extends over a considerable part of the MCMV and HCMV IE1 proteins (aa 33-500 and aa 23-479) (Fig. 2). However, it must be emphasized that the similarity between the aligned amino acid sequences is relatively low, with only 105 of the 468 amino acids in this region being identical (22\%) and another 20\% (95 aa) representing related residues. The expectation value calculated by the alignment program indicated that the similarity may not be significant. In contrast, the $88 \mathrm{kDa}$ MCMV IE3 (611 aa) 


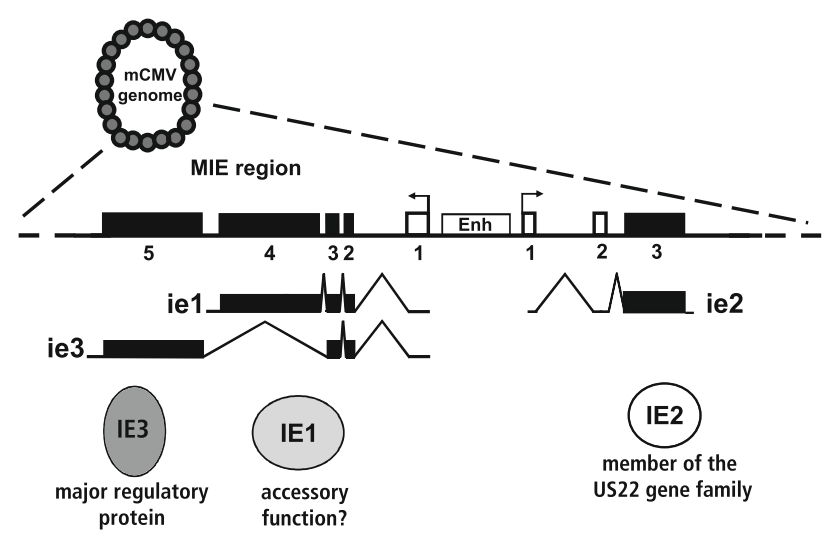

Fig. 1 Structural organization of the MCMV major immediate-early region. The two transcription units ie $1 / \mathrm{ie} 3$ and ie 2 are composed of several exons. Non-coding and coding exons are depicted as open and filled rectangles, respectively. Transcription in this region is controlled by the ie $1 /$ ie 3 and ie 2 promoters and the enhancer elements (Enh). The indicated transcripts give rise to the IE proteins of MCMV

and the $86 \mathrm{kDa}$ HCMV IE2 proteins (579 aa) are highly related, with $56 \%$ of the $\mathrm{C}$-terminal third of the amino acid sequences being identical or consisting of conserved residues. Based on the results of transient transfection assays the MCMV IE3 and HCMV IE2 proteins were identified as promiscuous transactivators of viral early promoters and as repressors of their own promoter (for review see [1-3]). The latter function suggests that these proteins are autoregulating their own expression. Furthermore, the HCMV IE2 protein is able to activate specific cellular promoters. The IE2 protein promotes the entry of cells into the cell cycle, but then it inhibits the synthesis of cellular DNA [4]. In this way, IE2 probably generates favorable conditions for replication of HCMV. Whether the MCMV IE3 protein has a similar impact on the cell cycle is not yet known.

Despite their limited similarity, highly related properties and functions have been described for the IE1 proteins of the CMVs (Table 1). An accessory function in the trans-
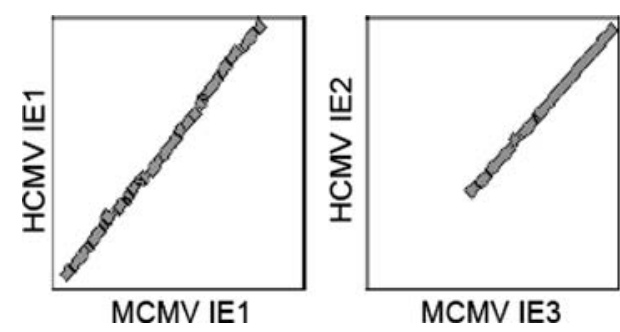

Fig. 2 Alignment of the amino acid sequences of the MCMV IE1 and IE3 proteins with their homologs in the HCMV AD169 strain and of the MCMV IE2 protein with related proteins of the rat CMV (English strain) and of HHV6A. Please note that the histograms indicate regions of similarity only and do not allow the quality of the alignments to be judged. The comparisons were done with the BLAST 2 SEQUENCES program [29] (using the following parameters, scoring matrix:
Table 1 Functions ascribed to the MCMV and HCMV IE1 proteins

\begin{tabular}{lll}
\hline Properties and functions & MCMV IE1 & HCMV IE1 \\
\hline Binding to chromatin & - & + \\
Modification by SUMO-1 & $\mathrm{NK}$ & + \\
Interaction with & & \\
HDAC-2 & + & $\mathrm{NK}$ \\
HDAC-3 & $\mathrm{NK}$ & + \\
STAT1 and STAT2 & $\mathrm{NK}$ & + \\
$\quad(\rightarrow$ inhibition of IFN signaling) & & \\
Daxx & + & - \\
PML $(\rightarrow$ ND10 dispersion) & + & + \\
p107 (Rb-related protein) & $\mathrm{NK}$ & + \\
$\quad(\rightarrow$ cell cycle progression) & & + \\
trans-activation of & & + \\
Cellular genes & + & \\
Viral genes & + & \\
\hline
\end{tabular}

$N K$ not known

activation of early promoters was ascribed to the IE1 proteins, enhancing the effects of the MCMV IE3 [3] and the HCMV IE2 protein (reviewed in [2]). For the HCMV IE1 protein a positive regulatory function on the major IE promoter was also observed [5-7]. Both IE1 proteins cause the trans-activation of promoters of cellular genes involved in nucleotide metabolism such as the human dihydrofolate reductase [8] and the mouse thymidylate synthase [9]. Another well-documented effect of both IE1 proteins is the dispersal of substructures in the nucleus of cells, the ND10 nuclear domains, which are thought to constitute an intrinsic cellular defense mechanism against DNA viruses (for a review see [10]). Nevels et al. [11] showed an interaction of the HCMV IE1 protein with histone deacetylase 3 (HDAC3) in infected fibroblasts and Tang and Maul [12] detected an interaction between the MCMV IE1 protein and HDAC2, Daxx and PML, which are all constituents of the ND10 structures. They could also show that the IE1 protein inhibited
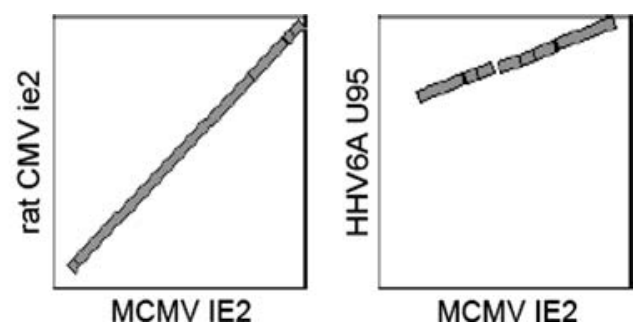

BLOSUM62, maximum length of gaps: 11, gap extension penalty: 1, word size: 3, x_dropoff: 50; expected significance: 10 ). Since the IE1 proteins contain many acidic residues, which will be classified as regions of low compositional complexity by the program, the filter function masking such residues was shut off for comparison of the IE1 proteins 
the deacetylation activity of HDAC-2. These data are consistent with the rescue of the growth impairment of an HCMV IE1 deletion mutant [13] at low multiplicities of infection (MOI) by HDAC inhibitors [11]. However, there seems to be no absolute requirement for the dispersal of ND10s for viral replication, because at high MOI, growth of the HCMV IE1 mutant is not impaired [13]. More recently it was reported that the HCMV IE1 protein is able to interfere with the effects of type I interferons by interacting with STAT1 and STAT2 [14]. This may at least partially explain the growth defect of the HCMV IE1 mutant at a low MOI, as the virus would no longer be able to restrict the host's antiviral defenses.

The genomic region on the other side of the enhancer elements is not conserved between MCMV and HCMV (Fig. 1). Remarkably however, there are homologs of the MCMV ie2 gene in other $\beta$-herpesviruses such as HHV6, HHV7 and the rat CMVs [15] (Fig. 2). This and the fact that sporadic transcription of the ie2 gene occurs during latency of MCMV [16] should encourage us to investigate the function of the MCMV IE2 protein, although in a previous study no obvious phenotype of an ie 2 mutant could be found in vitro or in vivo [17].

Many of the functions of IE genes of mouse and human CMV have been studied in transient assays using the isolated genes, or at best with HCMV mutants in cell culture. To verify the functions of the IE genes in the context of the viral infection and to investigate their role in the pathogenesis of the CMVs, IE mutants have to be analyzed in vitro and in vivo. In particular, for in vivo investigation of the role of the IE genes, MCMV is, in our opinion, the model of choice.

\section{The IE3 protein is a key regulatory protein of MCMV}

Based on the results of transient transfection assays it was assumed that the IE3 protein (similarly to its HCMV homo$\log$ IE2) has an important regulatory function in the very early phase of infection and possibly in later phases as well, but this had not been tested with viral mutants. The development of mutagenesis techniques applicable to bacterial artificial chromosome (BAC)-cloned CMV genomes greatly facilitated the construction of such mutants, because the generation of mutated genomes and the evaluation of the properties of these genomes and viruses derived from them, now represent separable and independent tasks [18, 19]. To assess the role of the ie 3 gene, a large deletion was introduced into exon 5, which disrupted the coding region for the IE3 protein, without affecting the neighboring ORFs [20] (Fig. 1). Upon transfection of this genome into mouse fibroblasts, plaque formation did not occur. To verify this result we also generated a mutant with a GFP reporter gene inserted into the exon 5-deleted genome at the transcription start site of the m128 (ie2) locus. In addition, a genome with a repaired ie 3 gene was constructed that carried the GFP gene at the same position. Whereas only single green fluorescing cells were observed following transfection of the $\Delta \mathrm{ie} 3$ genome, the repaired genome led to plaque formation indicative of productive infection. Similarly, co-transfection of the $\triangle \mathrm{ie} 3 \mathrm{BACs}$ and of a plasmid carrying the genomic region deleted in the mutant also resulted in plaque formation, most likely due to repair of the ie 3 gene by homologous recombination in the fibroblasts. These results let us conclude that the ie 3 gene is essential for growth of MCMV [20]. Still, we did not know at which stage the infection cycle of the $\Delta \mathrm{ie} 3$ virus was disrupted. To address this question, we had to generate a sufficient amount of the $\Delta \mathrm{ie} 3$ virus to perform infection experiments. To this end, complementing cell lines based on NIH 3T3 cells were generated, which provided the IE3 protein (and the IE1 protein) in trans. Different cell clones supported the replication of the $\Delta \mathrm{ie} 3$ mutant to varying degrees suggesting that an appropriate expression level of the IE proteins is crucial. Two cell clones turned out to be particularly suitable for generation of the $\Delta \mathrm{ie} 3$ viruses, although, depending on the inoculation dose, the final virus yields remained one to two orders of magnitude lower than the titers that could be achieved with the wild-type MCMV. The virus preparations occasionally contained a minor fraction of replicationcompetent viruses that probably originated from the repair of the ie 3 gene in the viral genomes by recombination with the viral sequences present in the genomes of the complementing cells. For further studies the complementing cell lines should be constructed in such a way that sequence overlaps that allow recombination and repair of the viral genome is avoided. However, due to the overlap with other transcription units as is the case with the ie 3 gene, this goal may not always be achievable. In addition, restoration of replication-competence due to non-homologous re-insertion of the missing sequences can probably never completely be prevented. For this reason, the resulting virus preparations have to be tested carefully for the absence of contamination with replication-competent viruses.

RT-PCR analysis of RNA from cells infected with the $\Delta$ ie3 mutants revealed that the ie1 transcript was synthesized in infected non-complementing cells, regardless of whether the infection was performed in the absence or presence of the protein synthesis inhibitor cycloheximide or whether RNAs were analyzed early or late during the infection cycle (Fig. 3) [20]. The ie3 transcript was of course missing, due to the disruption of the ie3 ORF. From another study, we know that the ie 2 transcript is also synthesized in $\Delta$ ie3-infected cells. The apparently higher level of ie1 transcripts in $\Delta$ ie3-infected cells (lane 3 ) in comparison to wild-type MCMV infected cells (lane 1; the ie1 signal is 


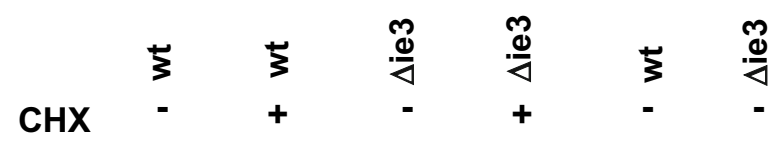

ie1

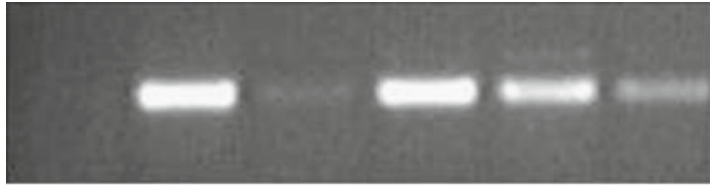

ie3

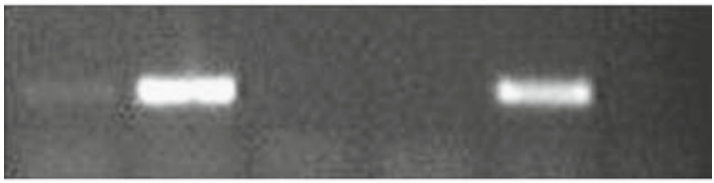

pol

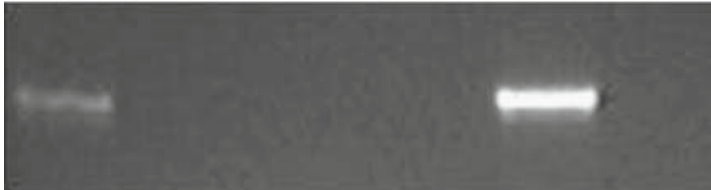

gB

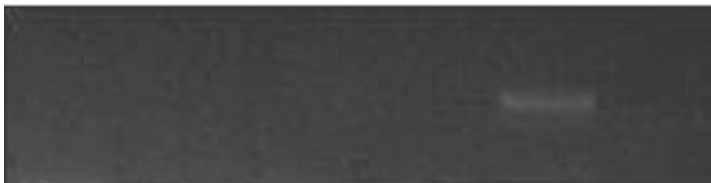

gL

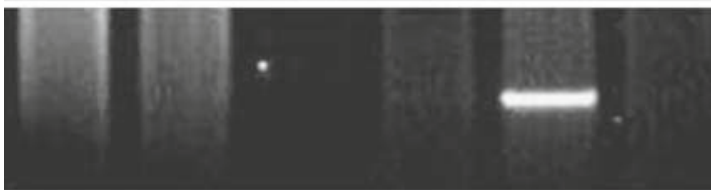

hprt

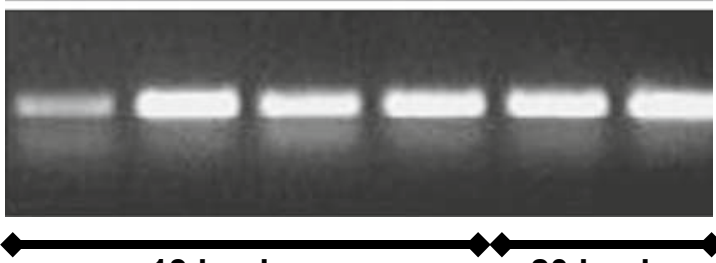

13 h p.i.

20 h p.i.

Fig. 3 Disruption of viral gene expression in cells infected with the $\Delta$ ie3 mutant. Transcripts representative of the IE (ie1, ie3), early [viral DNA polymerase (pol)] or late phase (glycoproteins $\mathrm{gB}$ and $\mathrm{gL}$ ) of MCMV were detected by RT-PCR in RNA isolated from cells that were infected with the wild-type MCMV (wt) or the $\triangle \mathrm{ie} 3$ mutant $(\Delta \mathrm{ie} 3)$ for the times indicated below. The signal originating from the cellular transcript hprt served as a loading control. Two samples were treated with cycloheximide $(+\mathrm{CHX})$ to restrict gene expression to IE transcription. Please note that the $\Delta \mathrm{ie} 3$ mutant expressed ie1 transcripts only, irrespective which conditions were used. The figure was reproduced from Ref. [20] with the permission of the American Society of Microbiology and the authors

below the detection limit in this specific experiment, possibly also due to a lower amount of RNA used) may result from the loss of the repression of the ie $1 /$ ie 3 promoter by the IE3 protein. Taken together, expression of the IE genes with the exception of the ie 3 gene does occur in $\Delta$ ie 3 infected cells. However, transcripts of early and late viral genes could never be detected. This result clearly indicated that the IE3 protein is at least needed for the activation of early gene transcription. Whether IE3 is also directly involved in the activation of late gene expression cannot be deduced from the infection experiments with the $\Delta$ ie 3 mutants as it could be that early proteins, which are not generated in $\Delta$ ie 3 infected cells, may be sufficient for the activation of late gene expression. However, in consideration of results obtained in transfection experiments, an important contribution of the IE3 protein in the activation of late genes is very likely. Later on, similar results were obtained with $\triangle \mathrm{IE} 2$ mutants of HCMV, indicating that the HCMV IE2 protein has a comparable role to the MCMV IE3 protein [21-23].

In order to determine the role of the ie 3 gene during the different phases of the lytic replication cycle and ideally also during reactivation of MCMV from latency, it was desirable to generate a mutant expressing a conditional variant of the IE3 protein. For this purpose we investigated whether the IE3 protein could be split into two separate subdomains, which were expressed as fusions with the FK506-binding protein (FKBP) and FKBP-rapamycin-associated protein (FRAP), and could be re-assembled as a functional IE3 molecule upon addition of an artificial dimerizer [24]. This approach led to promising results when trans-activation of the early viral e1 promoter by the split IE3 protein was tested in transient assays (B. Rupp, M. Messerle, unpublished results). However, replication-competent virus could not be obtained following insertion of the DNA sequences encoding the IE3 fusion proteins into the MCMV genome. In a similar approach, we fused a tamoxifen-inducible estrogen receptor domain [25] to the IE3 protein. This should cause the IE3 fusion protein to be sequestered in the cytoplasm in the absence of the inducer molecule and thus prevent the IE3induced transcription of early viral genes in the nucleus. The resulting virus was viable, however, the viral growth rate could be influenced by tamoxifen only marginally. Localization studies revealed that a substantial fraction of the IE3 fusion protein was transferred into the nucleus already in the absence of tamoxifen (B. Rupp, M. Messerle, unpublished results), perhaps because the nuclear localization signal in the IE3 amino acid sequence was overriding the capacity of the estrogen receptor domain to retain the fusion protein in the cytoplasm. The successful generation of a conditionally replicating HCMV mutant relying on a temperature-sensitive IE2 protein indicates that in principle such an approach is feasible [22]. We assume that a refinement of the above mentioned attempts will allow us to construct conditional ie3 mutants. Such tools would be highly valuable for dissecting the exact role of the IE3 protein in CMV pathogenesis.

\section{The MCMV ie1 deletion mutant is attenuated in vivo}

As the ie1 gene shares its first three exons with the ie 3 gene, we decided to delete the entire exon 4 to specifically 
destroy the reading frame for the $89 \mathrm{kDa}$ IE1 protein, leaving the IE3 ORF unaffected [26]. Exon 5 was directly fused to exon 3 promoting exclusive expression of the IE3 protein from this IE transcription unit in the $\Delta$ ie 1 mutant. We were aware that the expression of the IE3 protein may be regulated differently, since splicing from exon 3 to exon 5 is not required in this mutant. The complete elimination of exon 4 , however, prevented the usage of any cryptic splice site in this region and guaranteed that no aberrant IE1 product was generated, which could potentially substitute for the authentic $89 \mathrm{kDa}$ IE1 protein and distort the results. In order to confirm that the phenotype of the $\Delta \mathrm{ie} 1$ mutant is solely due to the lack of exon 4 , we also constructed a revertant virus in which the ie 1 gene was repaired. The $\Delta \mathrm{ie} 1$ virus and the revertant could be recovered from the mutated genomes in murine fibroblasts without the need for complementation. As expected there was no expression of the $89 \mathrm{kDa}$ IE1 protein in cells infected with the $\Delta \mathrm{ie} 1$ mutant. Remarkably, growth of the $\Delta$ ie 1 mutant in NIH $3 T 3$ cells was not impaired or delayed at low or at high MOI. Similarly, there were no significant differences between the growth rates of the $\Delta \mathrm{ie} 1$ mutant, the parental wild-type virus and the revertant virus in primary embryonic fibroblasts, endothelial and epithelial cell lines, or in peritoneal macrophages. Thus, at least in these cell types the $89 \mathrm{kDa}$ IE1 protein does not seem to be required for viral growth. Early and late gene expression as exemplified by the $\mathrm{m} 112 / \mathrm{m} 113$-encoded proteins seemed to be unaltered as was the expression of the IE3 protein; at best the amounts of the IE3 protein were slightly increased. These results differ from the phenotype of an HCMV IE1 mutant, which displayed an impaired growth capacity at low MOI and a reduced accumulation of a number of delayed-early proteins $[13,27]$. In this context, one should remember that another MCMV ie1 mutant that carried a frameshift mutation within exon 4 was also slightly impaired in its growth kinetics [18]. Further studies are needed to learn why different CMV ie1 mutants display diverse growth phenotypes.

One common property of CMV IE1 proteins is their capacity to disperse PML bodies in the nucleus of infected cells. In agreement with the results of Tang and Maul [12], we found that the $\Delta \mathrm{ie} 1$ mutant is unable to disrupt the PML bodies early in infection (Fig. 4c). Interestingly, the number of PML bodies in cells infected with the $\Delta \mathrm{ie} 1$ mutant clearly declined at late times of infection suggesting that (an) additional viral function(s) take over this function at the late time point. We also noted that in cells infected with the wild-type virus or the $\Delta \mathrm{ie} 1$ mutant at 15 and $24 \mathrm{~h}$ p.i., the PML antigen could be detected in small aggregates in the nucleus, including in the replication compartments (see Fig. 4d, e; [26]). Whether there is a specific function of these aggregates in the late phase of the replication cycle remains to be seen.
Finally, we investigated whether the $\Delta \mathrm{ie} 1$ mutant is able to replicate in vivo. When BALB/c mice were inoculated intraperitoneally with a dose of $1 \times 10^{6} \mathrm{PFU}$, the $\Delta \mathrm{ie} 1$ mutant could be detected in various organs for several days indicating that the virus was able to spread in the organism. The titers of the $\Delta \mathrm{ie} 1$ mutant were, however, approximately one to one and a half orders of magnitude lower than those of the wildtype or revertant virus. Thus, the replication capacity of the $\Delta$ ie1 mutant was clearly reduced in vivo. Notably, however, the $\Delta \mathrm{ie} 1$ mutant was able to reach and to replicate in the salivary glands, a property that many other attenuated MCMV mutants have lost. When immunocompromised SCID mice were infected with the wild-type virus and the $\Delta \mathrm{ie} 1$ mutant, differences in the titers in various organs were similar to those seen in infected BALB/c mice. We concluded therefore that differences in the titers cannot be explained by an increased sensitivity of the $\Delta \mathrm{iel}$ mutant to the adaptive immune response, as this is lacking in the SCID mice. Rather, the $\Delta \mathrm{ie} 1$ mutant may replicate more slowly than the wild-type virus in cell types that are important for spread of the virus in vivo, maybe in a cell type that we did not consider in our in vitro analysis. Another possibility is that the virus may be more susceptible to the innate immune response, e.g. to the action of NK cells or to the anti-viral activity of cytokines, as these defense mechanisms are intact in SCID mice.

An important aspect that remains to be investigated is the capacity of the $\Delta \mathrm{ie} 1$ mutant to establish latency and to reactivate. For these studies the reduced replication capacity of the $\Delta$ ie1 mutant in vivo has to be taken into account, since this may influence the efficiency of the mutant to establish latency i.e., infection with a replication-impaired virus probably leads to a reduced viral genome load in latently infected organs. The viral load in turn will affect the ability of the virus to reactivate as was previously shown by Reddehase et al. [28] several years ago. Preliminary results indicate that infection conditions can be found for the $\Delta \mathrm{ie} 1$ mutant which lead to loads of viral genomes in latently infected organs that are comparable to those seen in infection with the wild-type virus (A. Busche, unpublished results). Thus, the $\Delta \mathrm{ie} 1$ mutant seems to be able to establish latency and we expect to uncover in the near future whether it is also able to reactivate.

\section{Impact of the MCMV ie1 gene on interferon responsive gene expression}

For HCMV it was recently suggested that the IE1 protein at least partially counteracts the antiviral activity of type I interferons [14]. As a comparable function of the MCMV IE1 protein could explain the attenuation of the MCMV $\triangle \mathrm{ie} 1$ mutant, we asked whether the IE1 protein has an impact on the expression of interferon-stimulated genes. To this end, we used a luciferase reporter plasmid containing interferon 

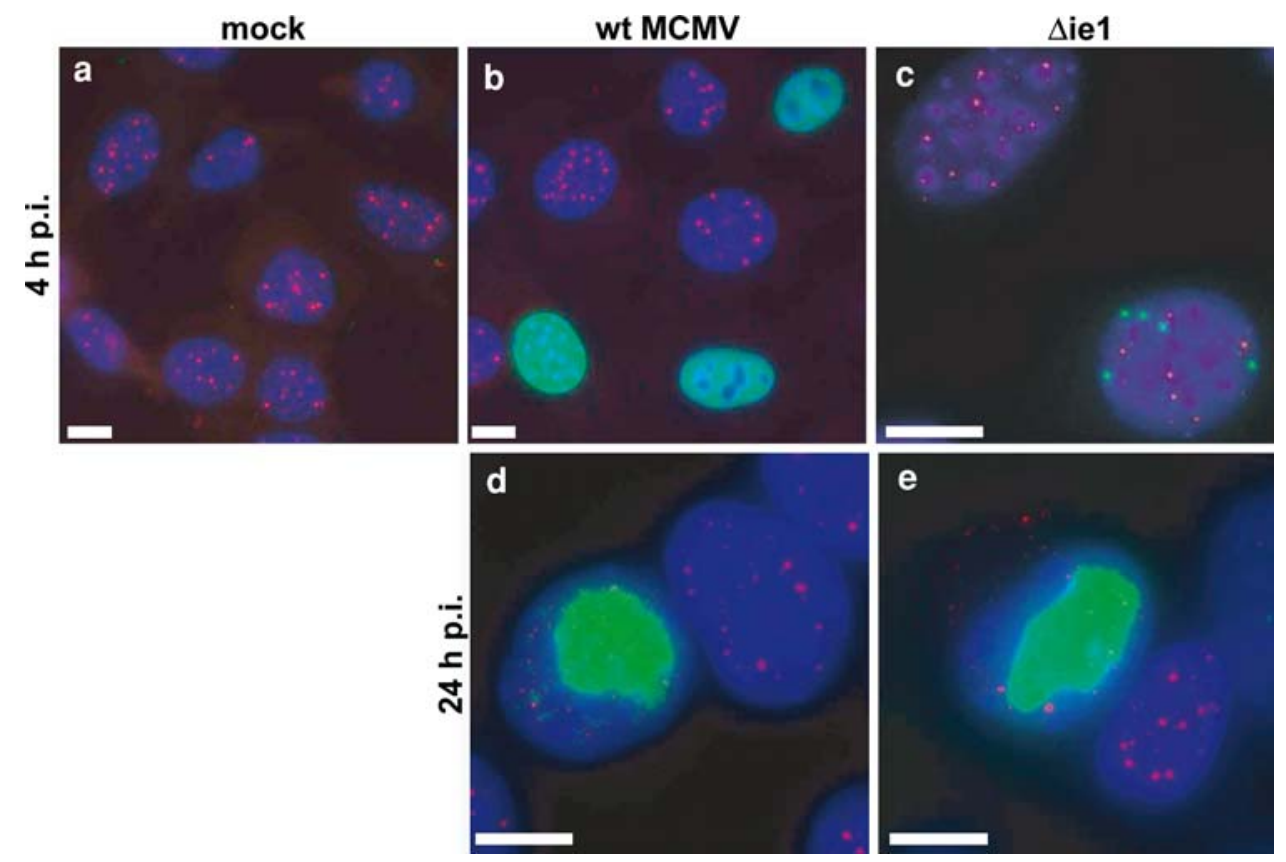

Fig. 4 Role of the MCMV IE1 protein in the dispersal of PML bodies. NIH 3 T3 cells were either mock treated or infected with wild-type MCMV or the $\Delta$ ie 1 mutant at an MOI of 0.3 and incubated for $4(\mathbf{a}-\mathbf{c})$ or $24 \mathrm{~h}(\mathbf{d}, \mathbf{e})$. Cells were fixed with paraformaldehyde and double labelled with MCMV IE1 or E1 and PML, and DNA was counterstained with DAPI (blue). Labeling of the PML bodies is in red. The IE1 protein (b) and the early viral protein E1 (c-e) are shown in green. Please note that the E1-specific antibody labels small pre-replication compart-

stimulated response elements (ISRE) and generated a cell line stably transfected with the construct. Following infection of this cell line, we detected a fourfold stronger induction of the promoter with the interferon stimulated response elements by the wild-type MCMV than the $\Delta \mathrm{ie} 1$ mutant (Fig. 5a). When the cells were stimulated with interferon- $\beta$ (IFN- $\beta$ ) and simultaneously infected with wild-type MCMV the ISRE trans-activation was more than twice as strong as after IFN- $\beta$ treatment only. The ISRE induction after treatment with IFN- $\beta$ and infection with the $\Delta$ iel mutant was in the same range as after treatment with IFN- $\beta$ alone, i.e., we did not observe an additional up-regulation as after infection with wild-type MCMV. A comparable induction profile was observed when cells were infected with wild-type MCMV or the $\Delta \mathrm{ie} 1$ mutant $2 \mathrm{~h}$ before interferon stimulation was applied (data not shown). Thus, our analysis suggested an activation of interferon-stimulated genes by the MCMV IE1 protein and there was definitely no inhibitory function of this MCMV IE protein on the expression of those genes at the early time point of $6 \mathrm{~h}$ p.i. as has been suggested for the HCMV IE1 homolog [14]. We next wanted to analyze whether the expression of viral genes is responsible for activating the transcription of the interferon inducible reporter gene. When the cells were treated with UV-inactivated MCMV virions, ISRE-driven expression was not induced ments at $4 \mathrm{~h}$ p.i. (c) and the large replication compartment at $24 \mathrm{~h} \mathrm{p.i.}$ (d, e). Late in infection, some PML bodies are retained in the nucleus of $\Delta$ ie1-infected cells (e), outside of the replication compartment. There are also some faint aggregates of PML in wild-type MCMV and $\Delta$ ie1-infected cells (d, e). Bars $10 \mu \mathrm{m}$. The figure was reproduced from Ref. [26] with the permission of the American Society of Microbiology and the authors

and when the cells were additionally treated with IFN- $\beta$ there was no additional activation beyond the induction that we already observed after IFN- $\beta$ treatment alone (Fig. 5b). This result strongly suggested that the IE1 protein is involved in the activation of the reporter gene. To investigate the capacity of the IE1 protein alone-independent of other viral proteins-to activate gene expression via the ISRE element murine fibroblasts were transiently cotransfected with an IE1 expression plasmid and the ISRE-driven luciferase reporter plasmid. While an unrelated control plasmid was unable to activate the interferon stimulated response element (ISRE), trans-activation by the ie 1 gene became apparent in a dose dependent manner (Fig. 5c). Taken together, these data suggest that the MCMV IE1 protein enhances rather than inhibits the trans-activation of the IFN type I stimulated response element. The observed activation by the IE1 protein is compatible with its proposed inhibitory activity on histone deacetylases [12]. Further experiments will reveal whether this mechanism can explain our results.

\section{Outlook}

A number of functions have been associated with the IE genes of CMVs. It is now well established that the IE3 protein of 

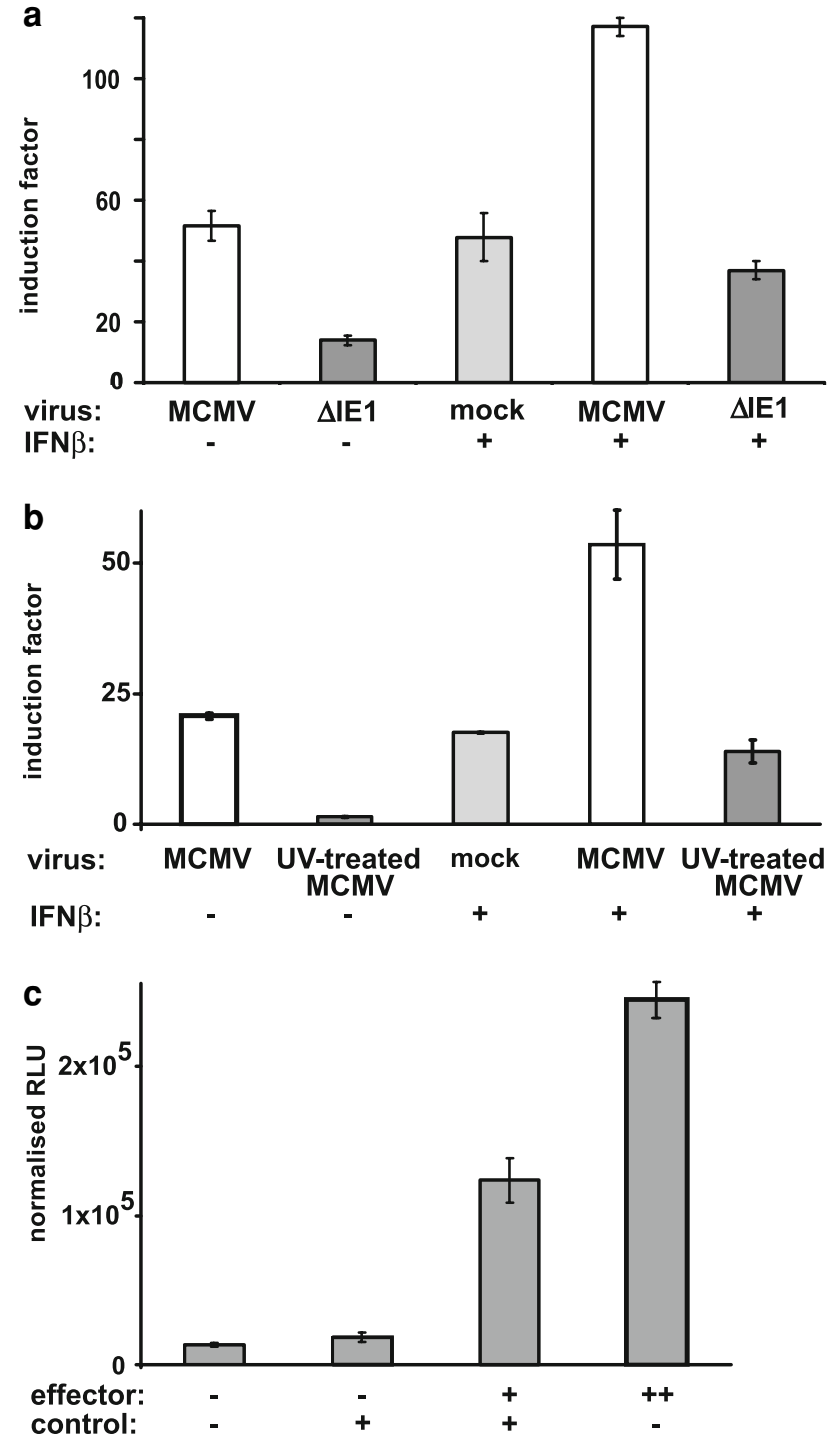

Fig. 5 Impact of the MCMV ie1 gene on interferon stimulated response element (ISRE)-controlled gene expression. a NIH 3T3 cells carrying a stably integrated ISRE-driven luciferase reporter construct were either mock-treated or infected with wild-type MCMV and the $\Delta$ ie1 mutant at an MOI of 2.5 and the luciferase activities were determined $6 \mathrm{~h}$ p.i. The induction factors depicted were calculated in relation to the luciferase activity obtained with the untreated samples. In a second series the cells were treated with 1,000 units $/ \mathrm{ml}$ of interferon- $\beta$ and then infected with the indicated viruses. $\mathbf{b}$ Viral gene expression is needed for stimulation of the ISRE. The reporter cells were treated with wild-type MCMV or UV-inactivated virus, or the cells were treated with interferon- $\beta$ and then infected as indicated. The luciferase activity was measured $6 \mathrm{~h}$ p.i. c Effect of the ie 1 gene in transient transfection assays. NIH 3 T3 cells were transfected with the ISRE-luciferase reporter plasmid $(1 \mu \mathrm{g})$, together with a control plasmid $(1 \mu \mathrm{g},[+])$ or with increasing amounts of the IE1-expressing construct $(1[+]$ and $2 \mu \mathrm{g}[++])$. Luciferase activities were determined $48 \mathrm{~h}$ p.i. and normalized to the $\beta$-galactosidase activities resulting from a co-transfected lacZ plasmid

MCMV and the IE2 protein of HCMV represent essential factors which, besides their other functions, act early in infection to trans-activate early viral and cellular genes. It is not yet clear whether these proteins are also needed for the trans-activation of late genes in order to maintain the lytic replication cycle. Similarly, we do not know whether the IE3 protein is essential for maintenance of latency. One may assume that either the recurrence of lytic infection has to occur occasionally to replenish the load of latent viral genomes or that the viral genomes are amplified from time to time, possibly by cellular enzymes, especially if the latently infected cells undergo cell division. The IE3 protein would be required at least in the first scenario. MCMV mutants with a conditional ie 3 gene, whose expression can be turned on and off at will, would be a highly useful tool to answer these questions.

The biological significance of the many functions ascribed to the IE1 proteins of MCMV and HCMV seems less clear at this time. The growth defect of HCMV IE1 mutants at low MOI has been associated with the ability of the IE1 protein to inhibit histone deacetylases as well as with its interference on the effects of type I interferons. Surprisingly, the MCMV $\triangle \mathrm{ie} 1$ mutant described here does not display growth impairment in cell culture and we saw no inhibition of interferon type I induced effects. Whether this is specific for this particular MCMV $\triangle \mathrm{ie} 1$ mutant or due to a general difference between the MCMV and HCMV IE1 proteins remains to be seen. The MCMV $\triangle \mathrm{ie} 1$ mutant is however attenuated in vivo. Currently, the underlying mechanism for the attenuation is not known, but this must be a function of the IE1 protein that is biologically relevant.

The knowledge of the interaction of the IE proteins with other viral and cellular proteins may allow us to design small compounds that interfere with or disrupt these interactions. If this can be achieved, we may be able to block the lytic replication of CMV or even the reactivation at a very early stage preventing the recurrence of $\mathrm{CMV}$ and the disastrous CMV disease in patients.

Acknowledgments Our work is supported by grants of the German Research Council (DFG; collaborative research grant 587, individual project A13 to M.M.), of the Ministerio de Ciencia y Tecnología (MCYT; SAF2005-05633 to A.A.) and of the Wellcome Trust (to P.G.).

\section{References}

1. Mocarski ES, Courcelle CT (2001) Cytomegaloviruses and their replication. In: Knipe DM, Howley PM (eds) Fields virology. Lippincott Williams \& Wilkins, Philadelphia, pp 2629-2674

2. Meier JL, Stinski MF (2006) Major immediate-early enhancer and its gene products. In: Reddehase MJ (ed) Cytomegaloviruses: molecular biology and immunology. Caister Academic Press, Wymondham, pp 151-166

3. Messerle M, Bühler B, Keil GM, Koszinowski UH (1992) Structural organization, expression, and functional characterization of the murine cytomegalovirus immediate-early gene 3 . J Virol 66:27-36

4. Wiebusch L, Hagemeier C (2001) The human cytomegalovirus immediate early 2 protein dissociates cellular DNA synthesis from cyclin-dependent kinase activation. EMBO J 20:1086-1098 
5. Stenberg RM, Stinski MF (1985) Autoregulation of the human cytomegalovirus major immediate-early gene. J Virol 56:676-682

6. Cherrington JM, Mocarski ES (1989) Human cytomegalovirus ie1 transactivates the alpha promoter-enhancer via an 18-base-pair repeat element. J Virol 63:1435-1440

7. Stenberg RM, Fortney J, Barlow SW, Magrane BP, Nelson JA, Ghazal P (1990) Promoter-specific trans activation and repression by human cytomegalovirus immediate-early proteins involves common and unique protein domains. J Virol 64:1556-1565

8. Margolis MJ, Pajovic S, Wong EL, Wade M, Jupp R, Nelson JA, Azizkhan JC (1995) Interaction of the 72-kilodalton human cytomegalovirus IE1 gene product with E2F1 coincides with E2Fdependent activation of dihydrofolate reductase transcription. J Virol 69:7759-7767

9. Gribaudo G, Riera L, Lembo D, De Andrea M, Gariglio M, Rudge TL, Johnson LF, Landolfo S (2000) Murine cytomegalovirus stimulates cellular thymidylate synthase gene expression in quiescent cells and requires the enzyme for replication. J Virol 74:4979-4987

10. Tang Q, Maul GG (2006) Immediate-early interactions and epigenetic defense mechanisms. In: Reddehase MJ (ed) Cytomegaloviruses: molecular biology and immunology. Caister Academic Press, Wymondham, pp 131-149

11. Nevels M, Paulus C, Shenk T (2004) Human cytomegalovirus immediate-early 1 protein facilitates viral replication by antagonizing histone deacetylation. Proc Natl Acad Sci U S A 101:17234-17239

12. Tang Q, Maul GG (2003) Mouse cytomegalovirus immediate-early protein 1 binds with host cell repressors to relieve suppressive effects on viral transcription and replication during lytic infection. J Virol 77:1357-1367

13. Greaves RF, Mocarski ES (1998) Defective growth correlates with reduced accumulation of a viral DNA replication protein after lowmultiplicity infection by a human cytomegalovirus ie1 mutant. $\mathbf{J}$ Virol 72:366-379

14. Paulus C, Krauss S, Nevels M (2006) A human cytomegalovirus antagonist of type I IFN-dependent signal transducer and activator of transcription signaling. Proc Natl Acad Sci U S A 103:3840-3845

15. Voigt S, Sandford GR, Hayward GS, Burns WH (2005) The English strain of rat cytomegalovirus (CMV) contains a novel captured CD200 (vOX2) gene and a spliced CC chemokine upstream from the major immediate-early region: further evidence for a separate evolutionary lineage from that of rat CMV Maastricht. J Gen Virol 86:263-274

16. Grzimek NK, Dreis D, Schmalz S, Reddehase MJ (2001) Random, asynchronous, and asymmetric transcriptional activity of enhanc- er-flanking major immediate-early genes ie $1 / 3$ and ie 2 during murine cytomegalovirus latency in the lungs. J Virol 75:2692-2705

17. Cardin RD, Abenes GB, Stoddart CA, Mocarski ES (1995) Murine cytomegalovirus IE2, an activator of gene expression, is dispensable for growth and latency in mice. Virology 209:236-241

18. Messerle M, Crnkovic I, Hammerschmidt W, Ziegler H, Koszinowski UH (1997) Cloning and mutagenesis of a herpesvirus genome as an infectious bacterial artificial chromosome. Proc Natl Acad Sci USA 94:14759-14763

19. Borst EM, Hahn G, Koszinowski UH, Messerle M (1999) Cloning of the human cytomegalovirus (HCMV) genome as an infectious bacterial artificial chromosome in Escherichia coli: a new approach for construction of HCMV mutants. J Virol 73:8320-8329

20. Angulo A, Ghazal P, Messerle M (2000) The major immediateearly gene ie 3 of mouse cytomegalovirus is essential for viral growth. J Virol 74:11129-11136

21. Marchini A, Liu H, Zhu H (2001) Human cytomegalovirus with IE-2 (UL122) deleted fails to express early lytic genes. J Virol 75:1870-1878

22. Heider JA, Bresnahan WA, Shenk TE (2002) Construction of a rationally designed human cytomegalovirus variant encoding a temperature-sensitive immediate-early 2 protein. Proc Natl Acad Sci U S A 99:3141-3146

23. White EA, Clark CL, Sanchez V, Spector DH (2004) Small internal deletions in the human cytomegalovirus IE2 gene result in nonviable recombinant viruses with differential defects in viral gene expression. J Virol 78:1817-1830

24. Pollock R, Clackson T (2002) Dimerizer-regulated gene expression. Curr Opin Biotechnol 13:459-467

25. Feil R, Wagner J, Metzger D, Chambon P (1997) Regulation of Cre recombinase activity by mutated estrogen receptor ligandbinding domains. Biochem Biophys Res Commun 237:752-757

26. Ghazal P, Visser AE, Gustems M, Garcia R, Borst EM, Sullivan K, Messerle M, Angulo A (2005) Elimination of ie1 significantly attenuates murine cytomegalovirus virulence but does not alter replicative capacity in cell culture. J Virol 79:7182-7194

27. Gawn JM, Greaves RF (2002) Absence of IE1 p72 protein function during low-multiplicity infection by human cytomegalovirus results in a broad block to viral delayed-early gene expression. J Virol 76:4441-4455

28. Reddehase MJ, Balthesen M, Rapp M, Jonjic S, Pavic I, Koszinowski UH (1994) The conditions of primary infection define the load of latent viral genome in organs and the risk of recurrent cytomegalovirus disease. J Exp Med 179:185-193

29. Tatusova TA, Madden TL (1999) BLAST 2 sequences, a new tool for comparing protein and nucleotide sequences. FEMS Microbiol Lett 174:247-250 